\title{
Solvation enhances the distinction between carboxylated armchair and zigzag single-wall carbon nanotubes (SWNT-COOH)
}

\author{
Tapas Kar ${ }^{\text {a,* }}$, Upendra Adhikari ${ }^{a, \dagger}$, Steve Scheiner ${ }^{\text {a }}$, Ajit K. Roy ${ }^{\text {b }}$, Renato L. T. Parreira \\ ${ }^{c}$, Pedro A. de S. Bergamo ${ }^{c}$, Giovanni F. Caramori ${ }^{\text {d }}$, Felipe, S. S. Schneider ${ }^{d}$ \\ ${ }^{a}$ Department of Chemistry and Biochemistry, Utah State University, Logan, UT 84322- \\ 0300, United States \\ ${ }^{\mathrm{b}}$ Materials and Manufacturing Directorate, Air Force Research Laboratory, Wright- \\ Patterson Air Force Base, Dayton, Ohio 45433, United States \\ ${ }^{\mathrm{c}}$ Núcleo de Pesquisas em Ciências Exatas e Tecnológicas, Universidade de Franca, \\ Franca, SP 14404-600, Brazil. \\ ${ }^{\mathrm{d}}$ Departamento de Química, Universidade Federal de Santa Catarina, Campus \\ Universitário Trindade, CP 476, Florianópolis, SC 88040-900, Brazil.
}

\begin{abstract}
The effect of various solvents on the structures and properties of carboxylated SWNTs has been explored using the Same Level Different Basis Set approach (SLDB), where B3LYP functional of density functional theory (DFT) was applied. Armchair $(4,4)$ and zigzag $(8,0)$ and $(9,0)$ tubes were considered as the testbed. In order to simulate varying concentration of $-\mathrm{COOH}$ groups, one to five acids groups were placed at one end of these tubes. These samples were placed in different solvents (namely, $\mathrm{CS}_{2}$, THF and water) with varying polarity and results were compared with gas-phase properties. Similar to the gas-phase, zigzag tubes also exhibit both regular $(\mathrm{r}-\mathrm{COOH}, v(\mathrm{C}=\mathrm{O})$ above $1700 \mathrm{~cm}^{-1}$ ) and low-frequency (lf-COOH, $v(\mathrm{C}=\mathrm{O})$ below $1700 \mathrm{~cm}^{-1}$ ) acid groups. Characteristics of r-COOH group are not affected much in solvents, but lf-COOH of the zigzag tube is the one that makes these tubes distinguishable from its armchair cousin. Stability and charge distribution of SWNT-COOH strongly depend on the number of acid groups in different solvents which may help controlling further functionalization. Vibrational analyses reveal certain features in the $1400-1600 \mathrm{~cm}^{-1}$ range that are characteristic of lf-COOH in different solvents, which may help in the assignment of experimental spectra of oxidized SWNT in solvents.

* Corresponding author: Fax: +1-435-797-3390

E-mail address: tapas.kar@usu.edu (T. Kar),

$\dagger$ Present address: Department of Chemistry, University of North Carolina at Chapel Hill, Chapel Hill, North Carolina 27599, United States
\end{abstract}




\section{Introduction}

Carboxylated single-wall carbon nanotubes (SWNT-COOH, o-SWNT), created during purification of raw SWNTs by oxidative treatments, are the gateway to the functionalized carbon nanotube arena. These functionalized nanotubes are a focus of nanotube research due to their wide range of applications, such as sensors, solar cells, drug delivery systems, and various electronic and optoelectronic devices [1-9]. Despite significant progress in chemical modification of purified carbon nanotubes, inhomogeneity in raw as well as in oxidized SWNTs represents a bottleneck in progress toward application oriented technological developments of individual nanotubes. Better understanding of structures and properties of individual o-SWNT might lead to selective and controlled functionalization. In addition, a major hurdle in separating SWNTs might be overcome by virtue of subtle differences, if any, among o-SWNTs. Of several oxygen-containing functional groups, $-\mathrm{COOH}$ is the most widely observed, located primarily at the tips and also at side-wall of SWNTs during various acid treatments. It is thus imperative to have a thorough knowledge of the structure and intrinsic properties of individual o-SWNTs, as those $-\mathrm{COOH}$ groups are used as the anchor for further functionalization. In recent days purification techniques have been developed to create short and defect-free SWNTs [10, 11] and thus to maintain tube structures intact, end-functionalization via functional groups would be a reasonable choice.

Our recent studies [12-14] revealed differences between oxidized armchair and zigzag tubes based on $\mathrm{COOH}$ groups at the terminus. A completely new $\mathrm{C}=\mathrm{O}$ stretching mode was found around $1650 \mathrm{~cm}^{-1}$ for some standalone $\mathrm{COOH}$ groups, but only at zigzag edges, which are designated as low-frequency $\mathrm{COOH}$ (lf-COOH) groups. It worth mentioning that lf-COOH groups are not connected by any $\mathrm{C}=\mathrm{O}-\mathrm{-H}-\mathrm{O} \mathrm{H}$-bond among themselves, even at higher concentration, due to unfavorable alignment of $\mathrm{C}=\mathrm{O}$ and $\mathrm{H}-\mathrm{O}$ units of nearby groups and the O--H-O bond angle is narrower than $100^{\circ}$ [15]. As this frequency range is typical of quinones or their derivatives, most of the previous experimental studies assigned the peak accordingly. However, our results indicate that such a peak may be due instead to these lf- $\mathrm{COOH}$ groups.

This conclusion from our previous investigations on a wide range of SWNT$\mathrm{COOH}$ was derived from gas-phase calculations. Since experiments [1, 2, 5, 16-24] showed varying solubility of functionalized SWNTs in different solvents, then the question arises as to how such a difference in $\mathrm{COOH}$ groups at the zigzag and armchair edges differ in different solvents. Also, the stability of o-SWNTs in different solvents may be a key factor dictating selective functionalization of individual kinds of tubes. Since there is no indication from experimental results about the solubility or stability of individual oxidized tube, as the samples of purified tubes obtained from any source and oxidation/purification methods are a mixture of different kinds of o-SWNTs, a theoretical investigation seems worthwhile on this topic. In the present study, we attempt to obtaibn some insights into the characteristics of acid groups at the edges of SWNTs in different solvents that may provide some new insight into the wet chemistry of carboxylated single wall carbon nanotubes. 


\section{Method of calculations}

Investigations on large systems like nanotubes, including calculation of vibrational frequencies, are computationally highly demanding and so require a protocol that is both accurate and feasible. Kar and coworkers have [25-28] shown the advantages of using the Same Level Different Basis (SLDB) prescription over other procedures, such as the widely used ONIOM $[29,30]$ method, for studying SWNTs and their chemical modifications. In fact, several other studies also indicated ONIOM is not a reliable method for studying functionalized nanotubes due to disruption of the $\pi$-network [31-34]. In the SLDB approach, atoms in defined active sites are provided with larger sets of basis functions, while smaller sets are applied to the remaining atoms. Previous studies [26, 27] demonstrated that the use of a larger basis set for a small number of atoms at active sites is sufficient to reproduce structure, energetics, and vibrational spectra for sidewall and end-functionalized nanotubes.

This DFT-SLDB technique was used throughout in the present study. In this protocol, $4 \mathrm{n}(2 \mathrm{~m})$ carbons at the functionalized site of $(\mathrm{n}, \mathrm{n})$ armchair (zigzag $(\mathrm{m}, 0))$ tubes and functional groups were treated with 6-31G*(O+) (where a set of diffuse sp-function was added for electronegative oxygen atoms) and the $3-21 \mathrm{G}$ basis was used for the remaining carbons. A larger basis set $\left(6-31 \mathrm{G}^{*}\right)$ was used for terminal hydrogens at the functionalized end and 3-21G for hydrogens at the other end of SWNTs. Thus, $6-31 \mathrm{G}^{*}$ is used for 16 carbons of $(4,4)$ and $(8,0)$ tubes, and 18 carbons of $(9,0)$ tube. Use of such a combined basis set reduces computational time by more than $50 \%$ for SCF steps and by more than $60 \%$ for vibrational steps, while reproducing properties quite accurately [14]. The B3LYP variant of density functional theory (DFT) $[35,36]$ was employed in order to include correlation effects. The accuracy of normal mode calculations using the B3LYP functional is sufficiently high and includes an optimal cost-to-benefit ratio [37-40].

The self-consistent reaction field (SCRF) approach [41] was employed in which recently developed SMD method by the Truhlar group [42] was used for all solvent calculations. This method has been recommended in calculating free energy $(\Delta \mathrm{G})$ of solvation [43]. Solvents used in this study include carbon disulfide $\left(\mathrm{CS}_{2}\right.$, dielectric constant $\varepsilon=2.6105$ ), tetrahydrofuran (THF, $\varepsilon=7.4257$ ) and water (W, $\varepsilon=78.3553$ ).

Because computed harmonic vibrational frequencies are typically slightly overestimated (even for more accurate methods, such as MP2, CCSD(T) etc, as well as for larger basis sets) a scale factor is commonly used to improve agreement with experimental spectra. For example, a value of 0.960 is recommended for B3LYP/6-31G* [44]. Since we are primarily interested in the spectra of attached functional groups, application of a scale factor of 0.96 was deemed a reasonable choice.

All calculations were performed using the Gaussian-09 [45] code. Theoretical

vibrational modes were analyzed using Chemcraft [46] program. Pristine nanotube models were obtained using TubeVBS software [47], and in the o-SWNTs models, 
$\mathrm{COOH}$ groups were positioned using Chemcraft [46], which was also used to generate oSWNTs figures for geometry analyses.

\section{Results and Discussions}

Three SWNT models are considered in the present study and those are: armchair $(4,4)$, semiconducting zigzag $(8,0)$ and metallic zigzag $(9,0)$. The former two molecular models of tubes contain 64 carbon atoms, and 72 carbons constitute the $(9,0)$ tube. In all cases, carbon atoms at the edges were saturated with appropriate numbers of hydrogen atoms, except of course those hosting acid groups. To mimic varying concentration of acid groups, 1, 3, 5 and 8 acid groups were considered at one tip of SWNTs, keeping another end fully saturated with hydrogen atoms. All geometries were fully optimized without any constraints in different solvents and the results are compared with gas-phase properties.

\subsection{Structure and Geometry}

Fully optimized geometries of $(4,4)-(\mathrm{COOH})_{\mathrm{n}},(8,0)-(\mathrm{COOH})_{\mathrm{n}}$ and $(9,0)-(\mathrm{COOH})_{\mathrm{n}} \quad(\mathrm{n}=$ 1, 3, 5 and 8) in water are depicted in Figs. 1 and 2. For the sake of comparison all structures of carboxylated-SWNTs in gas-phase, $\mathrm{CS}_{2}$, THF, and water are given in Figs. S1-S4 (Supporting information material). Key geometric parameters of o-SWNTs are summarized in Tables 1-3 $(\mathrm{n}=1,3$ and 5) and S5 $(\mathrm{n}=8)$. It may be noted that for $\mathrm{n}=8$, all carbons at the tip of $(4,4)$ and $(8,0)$ tubes are saturated with $\mathrm{COOH}$ groups. Such a high concentration of acid groups at the tip of the tube is most unlikely due to steric factors, and wash/dry process of the oxidized tube during purification process most likely eliminates several acid groups. It can be seen from these figures that the orientation of $\mathrm{COOH}$ group(s), irrespective of their numbers, at the tips of o-SWNTs found in the gasphase are almost unchanged in different solvents, i.e., practically no effect of solvents on the orientation of acid groups. (Similar structural arrangements of $\mathrm{COOH}$ groups at the edge of $(9,0)-(\mathrm{COOH})_{9}$ in gas-phase was also reported by Chelmecka et al. [48].)

On the other hand, bond lengths $(\mathrm{C}=\mathrm{O}, \mathrm{C}-\mathrm{O}$ and $\mathrm{C}-\mathrm{COOH})$ show progressive changes from gas-phase with varying polarity of the solvent. In the case of the armchair tube, $\mathrm{C}=\mathrm{O}$ distances are within $1.212-1.220 \AA$ in gas-phase and are elongated by about 0.01 $\AA$ in water, with an intermediate increase in $\mathrm{CS}_{2}$ and then in THF. As found in gas-phase, zigzag tubes also exhibit both $\mathrm{r}$ (regular) and lf (low-field) - $\mathrm{COOH}$ groups depending on the total number of acid groups contained therein. For $n=3$ all acid groups are lf. For $\mathrm{n}=5$, three in $(8,0)$ and four in $(9,0)$ tube are lf- $\mathrm{COOH}$, while the remainder are regular type. The $\mathrm{C}=\mathrm{O}$ bonds of the former group are longer than the latter by about $0.01 \AA$ in gas-phase and these differences widen to about $0.02 \AA$ in water, i.e., close to double that observed in the armchair tube. Graphical presentation in Fig. S6 clearly shows differences in $\mathrm{R}(\mathrm{C}=\mathrm{O})$ from gas-phase to different solvents. The carbonyl bond of lf$\mathrm{COOH}$ is stretched significantly compared to regular acid groups as the polarity of solvent increases. All such distances in regular COOH of o-SWNTs are within 1.210 to $1.230 \AA$ and these values are in the same range that found in benzoic acid, anthracene$\mathrm{COOH}$ (representative of zigzag tubes) and phenanthrene- $\mathrm{COOH}$ (smaller model of the armchair type). In contrast, $\mathrm{R}(\mathrm{C}=\mathrm{O})$ in unusual lf- $\mathrm{COOH}$ groups stretches (by about 0.05 
$\AA$ ) from gas-phase to water, where the longest distance (about $1.275 \AA$ ) in water is found in single carboxylated zigzag tubes.

In contrast to progressive elongation of $\mathrm{C}=\mathrm{O}$ bonds with increasing polarity of the solvent, changes in the C-O bond (in the second column of Tables 1-3 and S5) are found chirality dependent. In the armchair tube, such bonds shrink by about $0.01 \AA$, while for zigzag tubes a reverse trend is noted. In fact, such opposite trends in $\mathrm{C}$-O distance related to the characteristics of the $\mathrm{COOH}$ group: decreases for regular acid group and increases in lf- $\mathrm{COOH}$ groups, are observed even when both types of acid group are present in the same tube, as in $(\mathrm{m}, 0)-(\mathrm{COOH})_{\mathrm{n}}$ where $\mathrm{n}=5$ and 8 . Changes in $\mathrm{r}(\mathrm{O}-\mathrm{H})$ (in the range of 0.975 to $0.980 \AA$ ) are less sensitive to different media. Solvents bring tube and acid group closer together as $\mathrm{R}(\mathrm{C}-\mathrm{COOH})$ (shown in the last column of Tables 1-3) decreases. Such bonds in armchair tube are almost insensitive to solvent polarity, whereas changes are noticeable in zigzag tubes. In general, $\mathrm{R}(\mathrm{C}-\mathrm{COOH})$ distance are shortened in the range of 0.03 to $0.06 \AA$. Once again, it is the lf- $\mathrm{COOH}$ groups affected by more than regular acid groups.

In summary, solvents have noticeable effects (compared to gas-phase structures) on the key geometric parameters of carboxylated SWNTs. Such effects are more pronounced for lf-COOH groups than regular acid groups. The differences between two types of acid groups are progressively enhanced with the increasing polarity of the solvent. Such features may affect further functionalization of o-SWNTs in different solvents.

\subsection{Solvation energy}

The effects of different solvents on the stability of SWNTs and o-SWNTs are shown in Fig. 3. For the sake of comparison the stability of anthracene (simplest model of the zigzag tube) and phenanthrene (simplest model of the armchair tube), and their mono$\mathrm{COOH}$ derivatives are also included in the figure. The free energy of salvation $\left(\Delta \mathrm{G}_{\text {Solv }}\right)$ were estimated by the difference in energies in the gas-phase and in solvents, and a negative $\Delta \mathrm{G}_{\text {Solv }}$ value is an indication of stability compared to gas-phase. (It may be noted that stability in solvents is not necessarily directly correlated with an indicator of solubility.) Stability of carboxylated carbon networks in solvents is dictated by two opposing factors: destabilization due to hydrophobic carbon materials and stabilization by hydrophilic acid groups.

To estimate the stability of $-\mathrm{COOH}$ group itself in solvents, formic acid $(\mathrm{H}-$ $\mathrm{COOH}$ ) is considered as a model. Since in the models of o-SWNTs, acid groups are not interacting among themselves (such as O--H-O H-bonding), they may be considered as a standalone group at the tip of SWNTs. To model such multiple non-interacting acid groups (to represent 1,3 and 5 groups in o-SWNTs), $\Delta \mathrm{G}_{\text {Solv }}$ of $\mathrm{H}-\mathrm{COOH}$ (For-a) is multiplied by 3 ( $3 *$ For-a) and 5 ( $5 *$ For-a); changes in the stability with increasing number of $\mathrm{COOH}$ groups are shown in the top part of Fig. 3. As expected, $\Delta \mathrm{G}_{\mathrm{Solv}}$ of $(\mathrm{HCOOH})_{\mathrm{x}}(\mathrm{x}=1,3$ and 5) becomes progressively more negative in more polar solvent, i.e., the stability of formic acid enhances as solvent polarity increases. In contrast, the solvation energy of anthracene $\left(\mathrm{C}_{14} \mathrm{H}_{10}\right)$ and phenanthrene $\left(\mathrm{C}_{14} \mathrm{H}_{10}\right)$ decreases (shown in the top part of Fig. 3) as the polarity of solvent increases. Since both contain the same number of carbon and hydrogen atoms, effects of solvents on their solvation energies 
$\left(\Delta \mathrm{G}_{\text {Solv }}\right.$ ) are very close to one another. Due to the presence of polar $\mathrm{COOH}$ groups, their acidic forms $\left(\mathrm{C}_{14} \mathrm{H}_{9} \mathrm{COOH}\right)$ are found to be more stable than hydrocarbon structures and the difference $\left(\Delta \Delta \mathrm{G}_{\text {Solv }}\right)$ between $\Delta \mathrm{G}_{\text {Solv }}$ of hydrocarbon and acid is widened with increasing polarity of the solvents. For example, the $\Delta \Delta \mathrm{G}_{\text {Solv }}$ of about $2.0 \mathrm{kcal} / \mathrm{mol}$ in $\mathrm{CS}_{2}$ is multiplied by a factor of 4 in water.

It appears from the $\Delta \mathrm{G}_{\text {Solv }}$ (Fig. 3) that the effect of solvent is almost independent of the chirality of the tube but strongly depends on the number of carbon atoms (considering the negligible effect of terminal $\mathrm{H}$ atoms) as has been found in smaller models. As the number of carbon atoms increases from smaller models $\left(\mathrm{C}_{14}\right)$ to nanotubes $\left(\mathrm{C}_{64}\right.$ in $(8,0)$ and $(4,4)$, and $\mathrm{C}_{72}$ in $\left.(9,0)\right)$, the trend of destabilization with increasing polarity of solvent is more pronounced. The $\Delta \mathrm{G}_{\text {Solv }}$ curve of pristine $(8,0)$ runs parallel to and below that of the $(4,4)$ tube but with almost a constant difference of about 0.5 $\mathrm{kcal} / \mathrm{mol}$ in all solvents considered herein. As the number of carbon atoms increases from 64 to 72 as in $(9,0)$ tube, $\Delta \mathrm{G}_{\text {Solv }}$ increases in magnitude from about $-37.0 \mathrm{kcal} / \mathrm{mol}$ to $43.0 \mathrm{kcal} / \mathrm{mol}$ in $\mathrm{CS}_{2}$ and then decreases to about $18.0 \mathrm{kcal} / \mathrm{mol}$ in water. The difference in $\Delta \mathrm{G}_{\text {Solv }}$ between two zigzag tubes decreases from 5.0 (in $\mathrm{CS}_{2}$ ) to $3.4 \mathrm{kcal} / \mathrm{mol}$ (in water).

The destabilizing trend with increasing polarity of solvent continues to single carboxylated SWNTs. However, the $\Delta \Delta \mathrm{G}_{\text {Solv }}$ value of $(4,4)-1 \mathrm{a}$ and $(8,0)-1 \mathrm{a}$ widens progressively from $1.6 \mathrm{kcal} / \mathrm{mol}$ in $\mathrm{CS}_{2}$ to $9.2 \mathrm{kcal} / \mathrm{mol}$ in water, where the former tube is less stable in water. Compared to $(8,0)-\mathrm{COOH}$, the larger (based on diameter) $(9,0)$ $\mathrm{COOH}$ is found more stable in $\mathrm{CS}_{2}$ by $6.5 \mathrm{kcal} / \mathrm{mol}$ and this value decreases to 2.8 $\mathrm{kcal} / \mathrm{mol}$ in water. Interestingly, solvation energies of $(4,4)-(\mathrm{COOH})_{3}$ fall between $\Delta \mathrm{G}_{\text {Solv }}$ of $(8,0)-\mathrm{COOH}$ and $(9,0)-\mathrm{COOH}$. A gap of solvation energies between oxidized armchair and zigzag tubes diminishes as acid concentration increases. The $\Delta \mathrm{G}_{\text {Solv }}$ value of the $(4,4)-5 \mathrm{a}$ tube is closer to $(8,0)-3 \mathrm{a}$. The same number of $\mathrm{COOH}$ groups in the $(9,0)-3 \mathrm{a}$ tube exhibits higher stability by about $3.0 \mathrm{kcal} / \mathrm{mol}$ than the smaller zigzag tube in different solvents. The trend in $\Delta \mathrm{G}_{\text {Solv }}$ at these concentrations ( 3 for zigzag tube and 5 for the armchair tube) seems balanced between stabilizing and destabilizing factors of SWNT$\mathrm{COOH}$.

Increasing the number of $\mathrm{COOH}$ groups to 5 in zigzag tubes leads to a progressive increase in $\Delta \mathrm{G}_{\text {Solv }}$ as solvent polarity increases and are most stable in water. This trend clearly suggests the stabilization factor is dominating the destabilizing factor. We have not included results of SWNT- $(\mathrm{COOH})_{8}$ as such high concentration of acid groups at the tips of the tube is most unlikely due to steric factors, and the wash/dry process of oxidized tubes during purification process most likely eliminates several acid groups.

In summary, the stability of carboxylated SWNTs strongly depends on the number of acid groups present in the sample. At lower acid concentrations, o-SWNT destabilizes with increasing polarity of the solvent. At medium concentration, stability is to some extent constant and at higher concentration stability increases with the polarity of the solvent. A clear distinction between armchair and zigzag tube is that former type attains a similar stability as the latter but with a higher number of acid groups

\subsection{Charge distributions}

Natural population analyses (NPA) [49, 50] were performed to obtain charge distributions. The trends in the computed group charge of $\mathrm{COOH}$ units of SWNT- 
$(\mathrm{COOH})_{\mathrm{n}}$ are shown in Fig. 4, which plots the acid group(s) charge versus the Onsager function $\mathrm{F}_{\mathrm{o}}=(\varepsilon-1) /(\varepsilon+2)$ that relates $\varepsilon$ to the permanent dielectric constant of each solvent $[41,51]$. Note that $\mathrm{F}_{\mathrm{o}}$ is equal to zero for the gas phase wherein $\varepsilon=1$, and climbs to an asymptote of unity as the solvent becomes progressively more polar and $\varepsilon$ approaches $\infty$. The high value of $\varepsilon$ for water leads to a $F_{o}$ of 0.96 , rather close to the asymptotic limit of 1.0 .

Since the carboxylic acid group is an electron withdrawing group, it is expected that $\mathrm{COOH}$ groups may pull some density from the tube and possess some negative charge, making SWNT slightly positive. Fig. 4 shows two sets of data distinguishable from each other. The first set of group charges, shown in the top part of the figure, belong to the regular $\mathrm{COOH}(\mathrm{r}-\mathrm{COOH})$ groups and lie within +75 me to -75 me. The second set of charges represent lf- $\mathrm{COOH}$ groups and is much more negative in the range between 125 me and -850 me. For both kinds of acid groups, negative charge increases (more pronounced for lf- $\mathrm{COOH}$ groups) with increasing value of $\mathrm{F}_{\mathrm{o}}$, i.e., pulls more density from the tube as the polarity of solvent increases.

The electron withdrawing capacity of the acid group in carboxylated SWNTs seems dependent on their numbers. For example, the presence of one and three groups at the edge of the $(4,4)$ tube makes tube positively charged: +22 me in $(4,4)-\mathrm{COOH}$ and +31 me in $(4,4)(\mathrm{COOH})_{3}$ in gas phase (see first column of Table S7). The tube loses electrons progressively as solvent polarity increases. In water, these same quantities are +35 me and +80 me, respectively. By increasing $\mathrm{n}$ to five, a curious opposite trend is noted. The sum of five acid group charges of $(4,4)-(\mathrm{COOH})_{5}$ in the gas-phase is +89 me, rather than the negative quantity normally expected. With increasing polarity of the solvent, this value diminishes to +8 me in water. It can be seen that $\mathrm{q}(\mathrm{COOH})$ of $(4,4)-\mathrm{COOH}$ group in different media is very close to that of benzoic acid (Ben-1a), as the curve lies below than that of Anth-1a and Phen-1A by only a small margin.

Since zigzag tubes contain both regular and lf- $\mathrm{COOH}$ groups, their charges are plotted separately in the figure. The group charge of regular $\mathrm{COOH}$ follows the same trend observed as in the carboxylated armchair tube and simple aromatic molecules. For $(8,0)-(\mathrm{COOH})_{5}$, the curve runs parallel to that of its armchair counterpart with the same acid concentration and loses a small amount of electron density to the tube. Enlargement of tube diameter to $(9,0)$ causes a decrease of the positive group charge (Table S7) to almost half, in comparison to the smaller acidic $(8,0)$ tube.

In gas-phase, lf- $\mathrm{COOH}$ group charge of oxidized zigzag tubes ranges between 125 and -240 me, with slightly more negative values in the case of larger $(9,0)$ tube compared to $(8,0)$ tube. The effect of solvent is significant as all curves are displaced downward progressively with increasing polarity of the solvent. Again acid concentration plays a critical role in charge distribution. For example, group charge of about -400 me for one low-frequency acid group increases to about $-600 \mathrm{me}$. Such a difference is more pronounced in $(9,0)$ tube where an additional 425 me is transferred from the tube to acid groups when number of acid group increase from one to four. In all cases, the contribution from each lf- $\mathrm{COOH}$ to the total value is almost the same.

The molecular electrostatic potential (MEP), describing charge distribution in three dimensions of a molecule, of different SWNT- $(\mathrm{COOH})_{3}$ in gas and water is illustrated in Fig. 5. Blue and red regions in MEP maps indicate positive and negative potential, respectively. Intermediate potentials are shown in green and yellow colors in 
the figure. In gas-phase, all three maps shown on the left are very similar: red-region on carbonyl oxygen, blue on hydrogen of $\mathrm{COOH}$, and green (with slight yellow) area spans the entire tube and finally blue at the non-acidic edge. No noticeable changes in MEP are found when the carboxylated $(4,4)$ tube is placed in water. However, differences are prominent for zigzag tubes in water. The acidic edge shows deeper red-region spread close to the first layer of the carbon ring, followed by yellow-green regions and then greater blue area at the other edge. Part of this blue region at the edge is due to $\mathrm{C}-\mathrm{H}$ bonds, which are limited to hydrogen atoms in the gas phase, but expands to one layer of carbons. MEP maps of $(8,0)$ and $(9,0)$ also show some differences, where red and blue regions are more intense in the latter tube and occupy more space, diminishing green region to smaller area in the middle of the tube. This may be due to larger diameter of $(9,0)$ tube than $(8,0)$ tube.

In summary, charge distribution between acid group(s) and SWNT depends on the type of acid group, regular versus low-frequency, and also on the number of such groups. The presence of lf- $\mathrm{COOH}$ group at zigzag edge makes them distinguishable from oxidized armchair tubes. Results indicate that it may be possible to tune electron flow from the tube to acid groups, making the tube more electropositive, by increasing the number of acid groups, in addition to varying solvent polarity. Such variation is only possible in zigzag tubes, which makes them different from their armchair cousin.

\subsection{Vibrational analyses}

IR spectroscopy, along with other analytical techniques, has been routinely used for identification of acid group(s) in oxidized SWNT structures. Special emphasis is placed on calculating the IR spectra which offer the hope of allowing experimentalists to distinguish one sort of oxidized-SWNTs from another. And perhaps most importantly, the factors that lead to quite unique structural and spectroscopic features of the carboxyl group of SWNT-COOH are isolated and identified. Lorentzian broadening with fwhm of $20 \mathrm{~cm}^{-1}$ was applied to each spectrum (scaled by 0.96) to better simulate an experimental result.

The calculated spectra of SWNT- $(\mathrm{COOH})_{\mathrm{n}}(\mathrm{n}=5 ; \mathrm{SWNT}=(4,4),(8,0)$ and $(9,0))$ in different solvents along with gas-phase are superimposed in Figs 6 and 7. For the sake of clarity, spectra of acidic $(4,4)$ and $(8,0)$ are shown in Figure 6 , while Figure 7 summarizes results from $(8,0)$ and $(9,0)$ tubes. The data provide a clear picture of differences between metallic armchair and semiconducting zigzag, and metallic versus semiconducting zigzag oxidized tubes. For other SWNT-COOHs, having one and three acid groups, superimposed spectra are displayed in Figs. S8 and S9 for $n=1$ and Figs. $\mathrm{S} 10$ and $\mathrm{S} 11$ for $\mathrm{n}=3$. Scaled $\mathrm{C}=\mathrm{O}$ frequencies of all carboxylated SWNTs are summarized in Table 4, where regular and low-frequency modes for $\mathrm{n}=5$ in zigzag tubes are shown separately. In the case of one and three $\mathrm{COOH}$ groups of zigzag tubes, all acid groups fall in the low-frequency category.

The $\mathrm{C}=\mathrm{O}$ stretching frequency of each $-\mathrm{COOH}$ group in small aromatic systems shifts to lower values from gas-phase to solvents and progressively moves to lower frequency, lowest in water. For example, $v(\mathrm{C}=\mathrm{O})$ changes from 1719 (gas-phase) to 1705 $\left(\mathrm{CS}_{2}\right)$ to 1692 (THF) to 1620 (water). The same trend is found in the case of anthracene$\mathrm{COOH}$ and phenanthrene- $\mathrm{COOH}$ (Fig. S12) with a progressive increase in intensity as 
the solvent polarity increases. The structural difference between single carboxylated anthracene (zigzag model) and phenanthrene (armchair model) in different media has an insignificant influence on the $\mathrm{C}=\mathrm{O}$ vibrational mode, except slightly higher intensity in the former molecule.

Similar decreasing trends in carbonyl vibrational mode in different media have been found in extended carbon networks. Armchair tube with five $\mathrm{COOH}$ groups exhibits a single IR peak (solid lines in Fig. 6) in all media, and $v(C=O)$ shifts from 1718 $\mathrm{cm}^{-1}$ (gas phase) to 1632 (water) $\mathrm{cm}^{-1}$, with intermediate values of $1708 \mathrm{~cm}^{-1}$ in $\mathrm{CS}_{2}$ and then $1698 \mathrm{~cm}^{-1}$ in THF. In general, these $\mathrm{C}=\mathrm{O}$ modes are pure or constitute the major contribution. With the increase of Fo, intensity increases sharply to a maximum value in water. Results in Table 4, indicate the concentration of acid groups at $(4,4)$ has very little effect on $\mathrm{C}=\mathrm{O}$ mode, as the position of the peak shifts to the right side by about $10 \mathrm{~cm}^{-1}$ or less as the number of groups increases by a factor of two.

Two carbonyl peaks are exhibited by the zigzag $(8,0)-(\mathrm{COOH})_{5}$ tube (Fig. 6), where underlined values are associated with regular $\mathrm{COOH}$ group and others with If$\mathrm{COOH}$. The $\mathrm{C}=\mathrm{O}$ stretching frequency of regular $\mathrm{COOH}$ groups (two in this case) of the zigzag tube is higher by $24 \mathrm{~cm}^{-1}$ in gas-phase than the corresponding value in the armchair tube, and this difference diminishes to $1 \mathrm{~cm}^{-1}$ in aqueous solution. However, absorption in acidic armchair tube occurs at a higher intensity than in the corresponding zigzag tube.

The characteristics of lf- $\mathrm{COOH}$ of $(8,0)-(\mathrm{COOH})_{5}$ change with the polarity of the solvent. In the gas-phase and less polar solvents $\left(\mathrm{CS}_{2}\right.$ and THF), lf-COOH is represented by one peak in the $1680-1630 \mathrm{~cm}^{-1}$ region, but in water it is split into two intense peaks well below $1600 \mathrm{~cm}^{-1}$. These two modes at 1533 and $1468 \mathrm{~cm}^{-1}$ are a mixture of $\mathrm{C}=\mathrm{O}$ and $\mathrm{C}=\mathrm{C}$ stretching vibrations in addition to $\angle \mathrm{COH}$ and $\angle \mathrm{CCH}$ bending modes. It may be noted that in both cases, the $\mathrm{C}=\mathrm{O}$ modes are not dominating, but are identifiable as carbonyl stretching vibration, where the higher frequency mode has a slightly larger contribution from $\mathrm{C}=\mathrm{O}$ vibration. Single and triple $\mathrm{COOH}$ groups in the $(8,0)$ tube also exhibit two peaks (Figs. S8 and S10) in their aqueous solution and those peaks appear at 1551 and $1502 \mathrm{~cm}^{-1}$ in $(8,0)-\mathrm{COOH}$ and at 1531 and $1480 \mathrm{~cm}^{-1}$ in $(8,0)-(\mathrm{COOH})_{3}$. In general, the nanotube $\mathrm{C}=\mathrm{C}$ stretching mode appears in the $1600-1650 \mathrm{~cm}^{-1}$ range and due to conjugation with $\mathrm{COOH}$ group, both modes shift to lowers values.

Fig. 7 provides comparison between IR spectra of semiconducting and metallic zigzag tubes in the $1400-1800 \mathrm{~cm}^{-1}$ region. Besides the difference in their electronic properties, the metallic $(9,0)$ tube is slightly wider than the semiconducting tube and contains four lf- $\mathrm{COOH}$ group as compared to three in the $(8,0)$ tube. The $\mathrm{C}=\mathrm{O}$ mode of regular $\mathrm{COOH}$ group(s), underlined values in the figure, for both tubes are very close to each other. For example, gas-phase absorption for both tubes occurs at $1742 \mathrm{~cm}^{-1}$, and a difference of only about $2-3 \mathrm{~cm}^{-1}$ with similar intensity is found in solvents.

The major difference found in the $\mathrm{C}=\mathrm{O}$ mode of lf- $\mathrm{COOH}$ group(s) between $(8,0)$ and $(9,0)$ tubes is a change in intensity. In the gas-phase, $\mathrm{CS}_{2}$ and THF, the $v(\mathrm{C}=\mathrm{O})$ frequencies of the larger tube are slightly red-shifted by 2 to $15 \mathrm{~cm}^{-1}$ compared to its smaller counterpart and intensity enhances progressively from gas-phase to THF, almost double in THF. However, both peaks in aqueous solution of the larger tube are blueshifted by a small margin compared to the smaller tube. Similar differences are found between zigzag tubs with lower acid concentration. 
In summary, characteristics of IR spectra in the range of $1400-1800 \mathrm{~cm}^{-1}$ of carboxylated armchair and zigzag tubes depends on the solvent polarity. Although the regular $\mathrm{COOH}$ groups of different tubes exhibit similar behavior, lf- $\mathrm{COOH}$ groups display major differences between two kinds of tube. However, no distinguishable features of IR spectra were noted to differentiate between semiconducting and metallic zigzag tubes; minor changes observed are most likely due to the structural difference between them and acid concentration. The $\mathrm{O}-\mathrm{H}$ modes of regular acid groups appear in the $3480-3550 \mathrm{~cm}^{-1}$ range with low intensity. Similar to $\mathrm{C}=\mathrm{O}$ modes, acidic zigzag tubes also exhibit two O-H peaks, where those belonging to lf-COOH appear above $3510 \mathrm{~cm}^{-1}$ and others below this value. However, due to low intensity, the difference between these groups may not be distinguishable experimentally.

\section{Conclusions}

Functionalization of single-wall carbon nanotubes improves their solubility in different solvents which allow those involved in wet chemistry of these fascinating materials to develop application-oriented products in a wide range of fields. In this investigation, we have explored the structure, stability and properties of carboxylated-SWNTs, with varying concentration of acid group, in different solvents, namely $\mathrm{CS}_{2}, \mathrm{THF}$, and water. Such acid groups at the edge of tubes are the anchor to further functionalization, and thus play an important role in the chemistry of SWNTs.

Some of the $\mathrm{COOH}$ groups on the zigzag $(8,0)$ and $(9,0)$ tubes exhibit different characteristics than those in the armchair $(4,4)$. Such groups are termed low-frequency (lf) acids based on their carbonyl vibrational frequency, which appears below $1700 \mathrm{~cm}^{-1}$ in the gas-phase. Such lf-COOH groups make the difference between armchair and zigzag tubes, and the discrepancies widen with increasing polarity of the solvent.

Stability of SWNT- $(\mathrm{COOH})_{\mathrm{n}}$ in different media depends on the number of acid groups and number of carbon atoms. It is well known that as the number of carbon atoms increases, the solubility of aromatic acid group decreases. Thus, the stability of SWNT$\mathrm{COOH}$ depends on two factors: destabilizing by tube size and stabilizing by increasing number of acid groups. At lower concentration of $\mathrm{COOH}$ units, the stability of acidicSWNT decreases as the polarity of solvent increases, while at a higher concentration a reverse trend is followed.

Electron distribution in SWNT-COOH indicates armchair tube gains or loses a certain amount of electron density and the magnitude is not affected by the solvent. However, zigzag tubes progressively lose density to the acid group as solvent polarity increases. This trend suggests the possibility of controlling selective second functionalization of zigzag tubes by varying acid concentration and solvent.

We also provided IR spectra of different oxidized tubes in different media, where the most characteristic $\mathrm{C}=\mathrm{O}$ stretching vibration mode of the acid group is analyzed critically. While $(4,4)-\mathrm{COOH}$ exhibits a single $\mathrm{C}=\mathrm{O}$ peak in the standard region, two intense peaks are common in their zigzag cousins. The most striking feature is the shift of $\mathrm{C}=\mathrm{O}$ frequency of lf-COOH groups below $1650 \mathrm{~cm}^{-1}$ in solvents. In aqueous solution, this peak is red-shifted and split to an additional peak below $1600 \mathrm{~cm}^{-1}$. Thus, the IR spectra of zigzag-COOH tubes show an unusual feature in the $1450-1650 \mathrm{~cm}^{-1}$ range, where $\mathrm{C}=\mathrm{O}$ modes are coupled with $\mathrm{C}=\mathrm{C}$ stretch, in addition to $\mathrm{CCH}$ and $\mathrm{COH}$ bending 
modes. This may be due to conjugation effects between $\mathrm{C}=\mathrm{O}$ and $\mathrm{C}=\mathrm{C}$ bonds. These findings may be helpful in assigning IR peaks experimentally.

\section{Acknowledgements}

This project is financially supported by CAPES (Brazil - Science without Borders program, number: 88881.068346/2014-01) (to TK, RLTP and GFC). Computational facilities at University of Franca, Federal University of Santa Catarina, Utah State University and DoD HPC at AFRL (Dayton, USA) are gratefully acknowledged. GFC and FSSS thank CNPq, Brazil (grant 302408/2014-2) for the financial support. RLTP and PAdeSB thank FAPESP, Brazil (grant 2011/07623-8). 


\section{REFERENCES}

[1] Niyogi S, Hamon MA, Hu H, Zhao B, Bhowmick P, Sen R, et al. Chemistry of single-walled carbon nanotubes. Acc Chem Res. 2002;35:1105-13.

[2] Lin T, Bajpai V, Ji T, Dai L. Chemistry of carbon nanotubes. Aust J Chem. 2003;56:635-51.

[3] Basiuk VA, Basiuk (Golovataya-Dzhymbeeva) EV. Chemical Derivatization of Carbon nanotube tips. California: American Scientific Publishers; 2004.

[4] Banerjee S, Hemraj-Benny T, Wong SS. Covalent surface chemistry of singlewalled carbon nanotubes. Adv Mater. 2005;17:17-29.

[5] Tasis D, Tagmatarchis N, Bianco A, Prato M. Chemistry of Carbon Nanotubes. Chem Rev. 2006;106:1105-36.

[6] Kauffman DR, Star A. Carbon nanotube gas and vapor sensors. Angew Chem Int Ed. 2008;47:6550-70.

[7] Cao Q, Rogers JA. Ultrathin films of single-walled carbon nanotubes for electronic and sensors: A review of fundamental and applied aspects. Adv Mater. 2009;21:29-53.

[8] Lu F, Gu L, Meziani MJ, Wang X, Luo PG, Veca LM, et al. Advances in bioapplications of carbon nanotubes. Adv Mater. 2009;21:139-52.

[9] Tian B, Kempa TJ, Lieber CM. Single nanowire photovoltaics. Chem Soc Rev. 2009;38:16-24.

[10] Kamalasanan K, Gottardi R, Tan S, Chen Y, Godugu B, Rothstein S, et al. "ZeroDimensional" Single-Walled Carbon Nanotubes. Angew Chem Int Ed. 2013;52:11308-12.

[11] Diaz E, Velazquez CG, Ortiz JV, Saucedo D, Ortega G, Paniagua AM. Purification of single-wall carbon nanotubes by functionalization and ultracentrifugation. Asian J Chem. 2013;25:S17-S21.

[12] Kar T, Scheiner S, Patnaik SS, Bettinger HF, Roy AK. IR characterization of tipfunctionalized single-wall carbon nanotubes. J Phys Chem C. 2010;114:20955-61.

[13] Kar T, Scheiner S, Roy AK, Bettinger HF. Unusual low-frequency $\mathrm{C}=\mathrm{O}$ mode of $\mathrm{COOH}$ group can distinguish between carboxylated zigzzag and armchair nanotubes. J Phys Chem C. 2012;116:26072-83.

[14] Kar T, Scheiner S, Roy AK. Reliability of Approximate Methods to Study TipFunctionalized Single-Wall Carbon Nanotubes. J Phys Chem C. 2012;116:254016.

[15] Scheiner S. Hydrogen Bonding. A Theoretical Perspective. Oxford: Oxford University Press; 1997.

[16] Sun Y-P, Fu K, Lin Y, Huang W. Functionalized carbon nanotubes: properties and applications. Acc Chem Res. 2002;35:1096-104.

[17] Banerjee S, Khan MGC, Wong SS. Rational chemical strategies for carbon nanotube functionalization. Chem Eur J. 2003;9:1898-908.

[18] Haddon RC, Sippel J, Rinzler AG, Papadimitrakopoulos F. Purification and separation of carbon nanotubes. MRS Bulletin. 2004;29:252-9.

[19] Banerjee S, Benny-Hemraj T, Wong SS. Routes towards separating metallic and semiconducting nanotubes. J Nanosci Nanotech. 2005;5(6):841-55. 
[20] Nakashima N. Soluble carbon nanotubes: fundamentals and applications. Intern J Nanosci. 2005;4(1):119-37.

[21] Murakami H, Nakashima N. Soluble carbon nanotubes and their applications. J Nanosci Nanotech. 2006;6:16-27.

[22] Singh S, Kruse P. Carbon nanotube surface science. Int J Nanotechnol. 2008;5:900-29.

[23] Barman SN, Pan D, Vosgueritchian M, Zoombelt AP, Galli G, Bao Z. Dispersion of single walled carbon nanotubes in amidine solvents. Nanotechnology. 2012;23:344011.

[24] Huang YY, Terentjev EM. Dispersion of Carbon Nanotubes: Mixing, Sonication, Stabilization, and Composite Properties. Polymers. 2012;4:275-95.

[25] Kar T, Akdim B, Duan X, Pachter R. A theoretical study of functionalized singlewall carbon nanotubes: ONIOM calculations. Chem Phys Lett. 2004;392(1-3):17680 .

[26] Kar T, Akdim B, Duan X, Pachter R. Open-ended modified single-wall carbon nanotubes: A theoretical study of the effects of purification. Chem Phys Lett. 2006;423:126-30.

[27] Kar T, Scheiner S, Roy AK. The effect on acidity of size and shape of carboxylated single-wall carbon nanotubes. A DFT-SLDB study. Chem Phys Lett. 2008;460:225-9.

[28] Kar T, Bettinger HF, Scheiner S, Roy AK. Noncovalent $\pi-\pi$ Stacking and CH--- $\pi$ Interactions of Aromatics on the Surface of Single-Wall Carbon Nanotubes (SWNTs) - An MP2 Study. J Phys Chem C. 2008;112(50):20070-5.

[29] Maseras F, Morokuma K. IMOMM: A new integrated ab initio + molecular mechanics geometry optimization scheme of equilibrium structures and transition states. J Comput Chem. 1995;16:1170.

[30] Morokuma K. ONIOM and its applications to material chemistry and catalyses. Bull Korean Chem Soc. 2003;24(6):797-801.

[31] Montoya A, Mondragon F, Truong TN. Adsorption on carbomaceous surfaces: cost-effective computational strategies for quantum chemistry studies of aromatic systems. Carbon. 2002;40:1863-72.

[32] Yim WL, Lou ZF. A reexamination of the chemisorption and desorption of ozone on the exterior of a $(5,5)$ single-wall carbon nanotube. Chem Phys Lett. 2004;398:297-303.

[33] Chen Z, Nagase S, Hirsch A, Haddon RC, Thiel W, Schleyer PvR. Side-wall opening of single-walled carbon nanotubes (SWCNTs) by chemical modification: A critical theoretical study. Angew Chem Int Ed. 2004;43:1552-4.

[34] Vreven T, Thompson LM, Larkin SM, Kirker I, Bearpark MJ. Deconstructing the ONIOM Hessian: Investigating Method Combinations for Transition Structures. J Chem Theo Comp. 2012;8:4907-14.

[35] Becke AD. Density-functional thermochemistry. III. The role of exact exchange. J Chem Phys. 1993;98:5648-52.

[36] Lee C, Yang W, Parr RG. Development of the Colle-Salvetti correlation-energy formula into a functional of the electron density,. Phys, Rev B. 1988;37:785-9. 
[37] Stephens PJ, Devlin FJ, Chabalowski CF, Frisch MJ. Ab Initio Calculation of Vibrational Absorption and Circular Dichroism Spectra Using Density Functional Force Fields. J Phy Chem. 1994;98:11623-7.

[38] Scott AP, Radom L. Harmonic Vibrational Frequencies: An Evaluation of HartreeFock, Møller-Plesset, Quadratic Configuration Interaction, Density Functional Theory, and Semiempirical Scale Factors. J Phys Chem. 1996;100:16502-13.

[39] Cheeseman JR, Frisch MJ, Devlin FJ, Stephens PJ. Ab initio calculation of atomic axial tensors and vibrational rotational strengths using density functional theory. Chem Phys Letts. 1996;252:211-20.

[40] Devlin FJ, Finley JW, Stephens PJ, Frisch MJ. Ab Initio Calculation of Vibrational Absorption and Circular Dichroism Spectra Using Density Functional Force Fields: A Comparison of Local, Nonlocal, and Hybrid Density Functionals. J Phys Chem. 1995;99:16883-902.

[41] Onsager L. Electric Moments of Molecules in Liquids. J Am Chem Soc. 1936;58:1486.

[42] Marenich AV, Cramer CJ, Truhlar DG. Universal solvation model based on solute electron density and a continuum model of the solvent defined by the bulk dielectric constant and atomic surface tensions. J Phys Chem B. 2009;113:637896.

[43] Alongi KS, Shields GC. Theoretical Calculations of Acid Dissociation Constants: A Review Article. Netherlands: Elsevier BV; 2010.

[44] Johnson III RD. NIST Computational Chemistry Comparison and Benchmark Database, 2006, available from http://cccbdb.nist.gov/vibscalejust.asp. NIST Computational Chemistry Comparison and Benchmark Database 2006.

[45] Frisch MJ, Trucks GW, Schlegel HB, Scuseria GE, Robb MA, Cheeseman JR, et al. Gaussian09: Gaussian, Inc., Wallingford CT, USA; 2009.

[46] Zhurko GA. http://www.chemcraftprog.com.

[47] Veiga RGA, Tomanek D. TubeVBS (http://k.1asphost.com/tubeasp/tubevbs.html). 2007.

[48] Chelmecka E, Pasterny K, Kupka T, Stobinski L. DFT studies of COOH tipfunctionalized zigzag and armchair single wall carbon nanotubes. J Mol Model. 2012;18:2241-6.

[49] Reed AE, Weinhold F, Curtiss LA, Pochatko DJ. Natural bond orbital analysis of molecular interactions: Theoretical studies of binary complexes of $\mathrm{HF}, \mathrm{H}_{2} \mathrm{O}, \mathrm{NH}_{3}$, $\mathrm{N}_{2}, \mathrm{O}_{2}, \mathrm{~F}_{2}, \mathrm{CO}$, and $\mathrm{CO}_{2}$ with $\mathrm{HF}, \mathrm{H}_{2} \mathrm{O}$, and $\mathrm{NH}_{3}$. J Chem Phys. 1986;84:5687705.

[50] Reed AE, Curtiss LA, Weinhold F. Intermolecular interactions from a natural bond orbital, donor-acceptor viewpoint. Chem Rev. 1988;88:899-926.

[51] Wong MW, Frisch MJ, Wiberg KB. Solvent effects. 1. The mediation of electrostatic effects by solvents. J Am Chem Soc. 1991;113(13):4776-82. 


\section{Appendix A. Supplementary data}

Fig. S1 - Optimized structures of (4,4)- $\mathrm{COOH},(8,0)-\mathrm{COOH}$ and $(9,0)-\mathrm{COOH}$ in gas, CS2, THF and water. Distances are in $\AA$.

Fig. S2 - Optimized structures of $(4,4)-(\mathrm{COOH})_{3}$ and $(8,0)-(\mathrm{COOH})_{3}$ in gas, CS2, THF and water. Distances are in $\AA$.

Fig. S3 - Optimized structures of $(4,4)-(\mathrm{COOH})_{5}$ and $(8,0)-(\mathrm{COOH})_{5}$ in gas, CS2, THF and water. Distances are in $\AA$.

Fig. S4 - Optimized structures of $(4,4)-(\mathrm{COOH})_{5}$ and $(8,0)-(\mathrm{COOH})_{5}$ in gas, CS2, THF and water. Distances are in $\AA$.

Table S5. Key geometric parameters ${ }^{\mathrm{a}}$ of SWNT- $(\mathrm{COOH})_{8}$. Bond lengths are in $\AA$ Fig. S6 - Variation of $\mathrm{C}=\mathrm{O}$ distance in different medium. Onsager function $\mathrm{F}_{\mathrm{o}}=(\varepsilon-1) /(\varepsilon$ +2 ), where $\varepsilon$ refers to the dielectric constant of the medium. Ben, Anth and Phen stand for benzene, anthracene and phenanthrene, respectively.

Table S7. NPA group charges (in me) of $\mathrm{COOH}$ groups. Negative numbers indicate charge flow from SWNT to $\mathrm{COOH}$, otherwise from $\mathrm{COOH}$ to SWNT.

Fig. S8 - B3LYP IR spectra of $(4,4)-\mathrm{COOH}$ and $(8,0)-\mathrm{COOH}$ in different medium. FWHF is considered at $20 \mathrm{~cm}^{-1}$. Theoretical frequencies are scaled by 0.96 .

Fig. S9 - B3LYP IR spectra of $(8,0)-\mathrm{COOH}$ and $(9,0)-\mathrm{COOH}$ in different medium. FWHF is considered at $20 \mathrm{~cm}^{-1}$. Theoretical frequencies are scaled by 0.96 .

Fig. S10 - B3LYP IR spectra of $(4,4)-(\mathrm{COOH})_{3}$ and $(8,0)-(\mathrm{COOH})_{3}$ in different medium. FWHF is considered at $20 \mathrm{~cm}^{-1}$. Theoretical frequencies are scaled by 0.96 .

Fig. S11 - B3LYP IR spectra of $(8,0)-(\mathrm{COOH})_{3}$ and $(9,0)-(\mathrm{COOH})_{3}$ in different medium. FWHF is considered at $20 \mathrm{~cm}^{-1}$. Theoretical frequencies are scaled by 0.96 . 
Figures Caption:

Fig. 1 - Optimized structures of $(4,4)-(\mathrm{COOH})_{\mathrm{n}},(8,0)-(\mathrm{COOH})_{\mathrm{n}}$ and $(9,0)-(\mathrm{COOH})_{\mathrm{n}}$ ( $n=1$ and 3 ) in water. Distances are in $\AA$. Optimized structures in other solvents and in gas-phase are given in Figures $\mathrm{S} 1(\mathrm{n}=1)$ and $\mathrm{S} 2(\mathrm{n}=3)$.

Fig. 2 - Optimized structures of $(4,4)-(\mathrm{COOH})_{n}$ and $(8,0)-(\mathrm{COOH})_{n}(\mathrm{n}=5$ and 8$)$ in water. Distances are in $\AA$. Low-frequency $\mathrm{COOH}$ groups are marked by lf. Optimized structures in other solvents and in gas-phase are given in Figures $S 3(n=5)$ and $S 4(n=8)$.

Fig. 3 - Variation of $\Delta \mathrm{G}_{\text {Solv }}$ of SWNT- $(\mathrm{COOH})_{\mathrm{n}}$ and simple organic acids in different medium. Onsager function $\mathrm{F}_{\mathrm{o}}=(\varepsilon-1) /(\varepsilon+2)$, where $\varepsilon$ refers to the dielectric constant of the medium. Ben, Anth, Phen and For stand for benzene, anthracene, phenanthrene and formic acid, respectively.

Fig. 4 - Variation of natural charge (q, me) of acid group of SWNT- $(\mathrm{COOH})_{\mathrm{n}}(\mathrm{n}=1,3$ and 5) and simple organic acids. If and $r$ stand for low-frequency and regular $\mathrm{COOH}$ group, respectively.

Fig. 5 - Top and side view of the molecular electrostatic potentials (MEP) of different SWNT- $(\mathrm{COOH})_{3}$ in gas-phase and in water.

Fig. 6 - B3LYP IR spectra of $(4,4)-(\mathrm{COOH})_{5}$ and $(8,0)-(\mathrm{COOH})_{5}$ in different medium. FWHF is considered at $20 \mathrm{~cm}^{-1}$. Theoretical frequencies are scaled by 0.96 . Underlined values are from regular $\mathrm{COOH}(\mathrm{r}-\mathrm{COOH})$ group of zigzag tube.

Fig. 7 - B3LYP IR spectra of $(8,0)-(\mathrm{COOH})_{5}$ and $(9,0)-(\mathrm{COOH})_{5}$ in different medium. FWHF is considered at $20 \mathrm{~cm}^{-1}$. Theoretical frequencies are scaled by 0.96 . Underlined values are from regular $\mathrm{COOH}$ group. 
Table 1. Key geometric parameters of SWNT-COOH. Bond lengths are in $\AA$.

\begin{tabular}{|l|l|l|l|l|}
\hline & $\mathrm{R}(\mathrm{C}=\mathrm{O})$ & $\mathrm{R}(\mathrm{C}-\mathrm{O})$ & $\mathrm{R}(\mathrm{O}-\mathrm{H})$ & $\mathrm{R}(\mathrm{C}-\mathrm{COOH})$ \\
\hline$(4,4)-\mathrm{COOH}$ & & & & \\
\hline Gas & 1.220 & 1.361 & 0.976 & 1.481 \\
\hline $\mathrm{CS}_{2}$ & 1.222 & 1.356 & 0.978 & 1.481 \\
\hline THF & 1.222 & 1.355 & 0.979 & 1.481 \\
\hline Water & 1.229 & 1.352 & 0.979 & 1.477 \\
\hline & & & & \\
\hline$(8,0)-\mathrm{COOH}$ & & & & \\
\hline Gas & 1.232 & 1.376 & 0.975 & 1.447 \\
\hline $\mathrm{CS}_{2}$ & 1.238 & 1.378 & 0.975 & 1.434 \\
\hline THF & 1.246 & 1.387 & 0.976 & 1.419 \\
\hline Water & 1.274 & 1.403 & 0.975 & 1.387 \\
\hline & & & & \\
\hline$(9,0)-\mathrm{COOH}$ & & & & \\
\hline Gas & 1.235 & 1.381 & 0.974 & 1.439 \\
\hline $\mathrm{CS}$ & 1.242 & 1.384 & 0.975 & 1.427 \\
\hline THF & 1.246 & 1.386 & 0.976 & 1.421 \\
\hline Water & 1.268 & 1.397 & 0.975 & 1.397 \\
\hline
\end{tabular}

Table 2. Key geometric parameters of SWNT- $(\mathrm{COOH})_{3}$. Bond lengths are in $\AA$

\begin{tabular}{|l|l|l|l|l|}
\hline & $\mathrm{R}(\mathrm{C}=\mathrm{O})$ & $\mathrm{R}(\mathrm{C}-\mathrm{O})$ & $\mathrm{R}(\mathrm{O}-\mathrm{H})$ & $\mathrm{R}(\mathrm{C}-\mathrm{COOH})$ \\
\hline$(4,4)-(\mathrm{COOH})_{3}$ & & & & \\
\hline Gas & 1.218 & 1.360 & 0.976 & 1.483 \\
\hline $\mathrm{CS}_{2}$ & 1.220 & 1.356 & 0.978 & 1.482 \\
\hline THF & 1.221 & 1.354 & 0.979 & 1.482 \\
\hline Water & 1.228 & 1.351 & 0.979 & 1.479 \\
\hline & & & & \\
\hline$(8,0)-(\mathrm{COOH})_{3}$ & & & & \\
\hline Gas & 1.227 & 1.371 & 0.975 & 1.457 \\
\hline CS $_{2}$ & 1.232 & 1.372 & 0.975 & 1.449 \\
\hline THF & 1.235 & 1.372 & 0.977 & 1.445 \\
\hline Water & 1.249 & 1.377 & 0.977 & 1.429 \\
\hline & & & & \\
\hline$(9,0)-(\mathrm{COOH})_{3}$ & & & & \\
\hline Gas & 1.227 & 1.371 & 0.975 & 1.459 \\
\hline CS & 1.232 & 1.369 & 0.976 & 1.369 \\
\hline THF & 1.237 & 1.373 & 0.977 & 1.443 \\
\hline Water & 1.258 & 1.387 & 0.976 & 1.415 \\
\hline
\end{tabular}


Table 3 Key geometric parameters ${ }^{\text {a }}$ of SWNT- $(\mathrm{COOH})_{5}$. Bond lengths are in $\AA$

\begin{tabular}{|l|l|l|l|l|}
\hline & $\mathrm{R}(\mathrm{C}=\mathrm{O})$ & $\mathrm{R}(\mathrm{C}-\mathrm{O})$ & $\mathrm{R}(\mathrm{O}-\mathrm{H})$ & $\mathrm{R}(\mathrm{C}-\mathrm{COOH})$ \\
\hline$(4,4)-(\mathrm{COOH})_{5}$ & & & & \\
\hline Gas $_{2}$ & $1.212-1.219$ & $1.347-1.360$ & $0.975-0.977$ & $1.480-1.508$ \\
\hline THF & $1.213-1.221$ & $1.343-1.355$ & $0.977-0.978$ & $1.480-1.509$ \\
\hline Water & $1.215-1.222$ & $1.342-1.354$ & $0.978-0.979$ & $1.480-1.508$ \\
\hline & $1.221-1.229$ & $1.338-1.351$ & $0.978-0.980$ & $1.479-1.508$ \\
\hline$(8,0)-(\mathrm{COOH})_{5}{ }^{\mathrm{b}}$ & & & & \\
\hline Gas & $1.210,1.214$ & $1.353,1.354$ & 0.977 & $1.498,1.503$ \\
& $1.223-1.226$ & $1.364-1.371$ & 0.975 & $1.454-1.460$ \\
\hline $\mathrm{CS}_{2}$ & $1.214,1.215$ & $1.348,1.351$ & 0.979 & $1.499,1.501$ \\
& $1.228-1.232$ & $1.365-1.370$ & 0.976 & $1.446-1.499$ \\
\hline THF & $1.216,1,217$ & $1.499,1.351$ & 0.980 & $1.498,1.499$ \\
& $1.232-1.236$ & $1.368-1.372$ & 0.977 & $1.440-1.450$ \\
\hline Water & $1.223,1.225$ & $1.348,1.348$ & 0.980 & $1.496,1.497$ \\
& $1.244-1.250$ & $1.375-1.379$ & 0.977 & $1.421-1.439$ \\
\hline & & & & \\
\hline$(9,0)-(\mathrm{COOH})_{5}{ }^{\mathrm{b}}$ & & & & \\
\hline Gas & 1.210 & 1.354 & 0.977 & 1.503 \\
& $1.224-1.227$ & $1.361-1.370$ & 0.975 & $1.456-1.473$ \\
\hline CS $_{2}$ & 1.213 & 1.350 & 0.979 & 1.502 \\
& $1.227-1.233$ & $1.359-1.370$ & 0.976 & $1.447-1.468$ \\
\hline THF & 1.217 & 1.350 & 0.980 & 1.499 \\
& $1.233-1.238$ & $1.366-1.371$ & 0.977 & $1.436-1.453$ \\
\hline Water & 1.226 & 1.346 & 0.980 & 1.495 \\
& $1.246-1.255$ & $1.37-1.381$ & 0.977 & $1.416-1.433$ \\
\hline
\end{tabular}

${ }^{a}$ Range of bond distances, ${ }^{b}$ The first set of bond lengths correspond to two regular (r) $\mathrm{COOH}$ groups and the second set of values are for three low-frequency (lf) $\mathrm{COOH}$ groups, see Fig. 2. (and S3) for positions of acid groups. Single distance is given when such bond lengths are same for all $-\mathrm{COOH}$ of the same group. 
Table 4. Scaled $\mathrm{C}=\mathrm{O}$ frequencies (in $\mathrm{cm}^{-1}$ ). $\mathrm{r}$ and lf stand for regular and low-frequency acid groups, respectively. 1a, $3 \mathrm{a}$ and $5 \mathrm{a}$ indicates number of acid groups. Number of $\mathrm{r}$ and lf- $\mathrm{COOH}$ group is given in parenthesis for zigzag- $(\mathrm{COOH})_{5}$.

$\begin{array}{llllll}(4,4)-1 a(r) & 1704 & 1692 & 1683 & 1614 & \\ (8,0)-1 a(\text { If) } & 1639 & 1606 & 1576 & 1551 & 1502 \\ (9,0)-1 a(\text { If) } & 1627 & 1608 & 1592 & 1520 & 1467 \\ & & & & & \\ (4,4)-3 a(r) & 1714 & 1701 & 1690 & 1620 & \\ (8,0)-3 a(3 \text { If) } & 1662 & 1638 & 1619 & 1531 & 1480 \\ (9,0)-3 a(3 \text { If) } & 1660 & 1636 & 1608 & 1533 & 1444 \\ & & & & & \\ (4,4)-5 a(r) & 1718 & 1708 & 1698 & 1632 & \\ (8,0)-5 a(2 \text { r) } & 1742 & 1724 & 1707 & 1633 & \\ (8,0)-5 a(3 \text { If) } & 1672 & 1649 & 1629 & 1533 & 1468 \\ (9,0)-5 a(1 \text { r) } & 1742 & 1726 & 1705 & 1630 & \\ (9,0)-5 a(4 \text { If) } & 1670 & 1634 & 1621 & 1545 & 1474\end{array}$




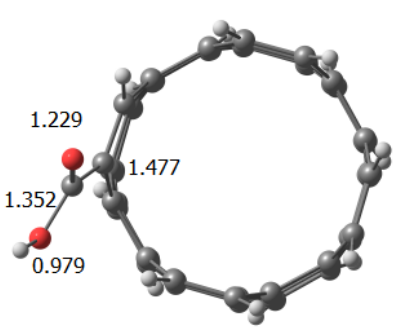

$(4,4)-1 a-w a t e r$

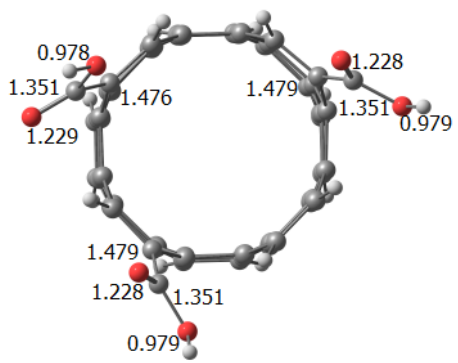

$(4,4)-3 a-w a t e r$

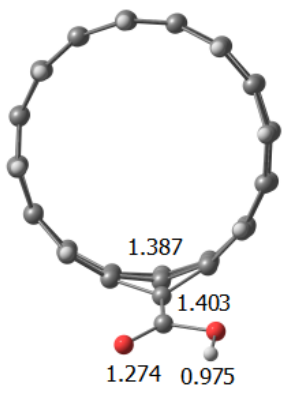

$(8,0)$-1a-water

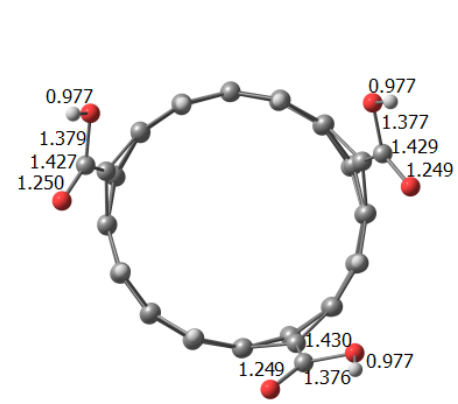

$(8,0)$-3a-water

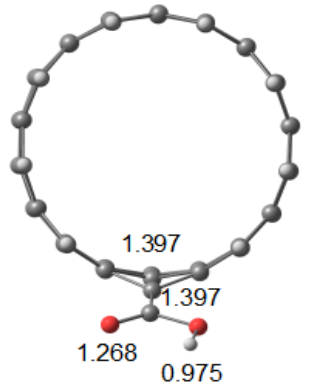

$(9,0)-1 a-w a t e r$

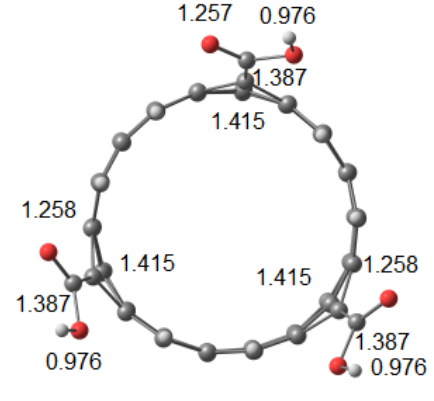

$(9,0)-3 a-w a t e r$

Fig. 1 - Optimized structures of $(4,4)-(\mathrm{COOH})_{\mathrm{n}},(8,0)-(\mathrm{COOH})_{\mathrm{n}}$ and $(9,0)-(\mathrm{COOH})_{\mathrm{n}}$ ( $n=1$ and 3 ) in water. Distances are in $\AA$. Optimized structures in other solvents and in gas-phase are given in Figures $S 1(n=1)$ and $S 2(n=3)$. 


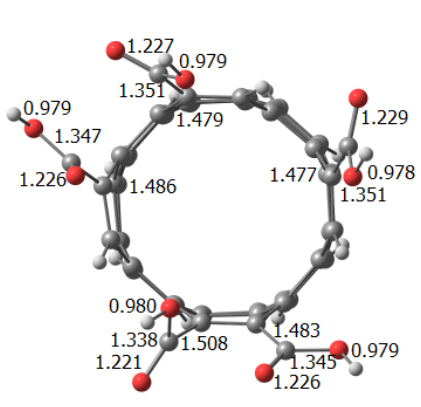

$(4,4)-5 a-w a t e r$

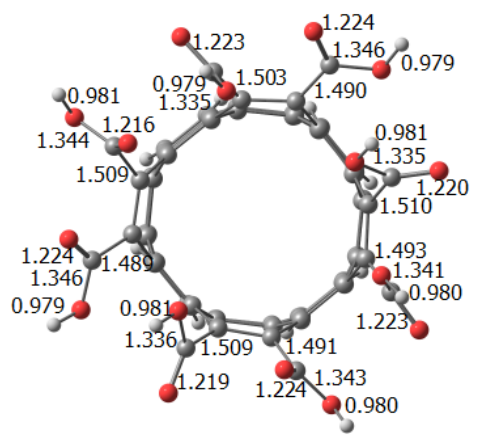

$(4,4)-8 a-w a t e r$

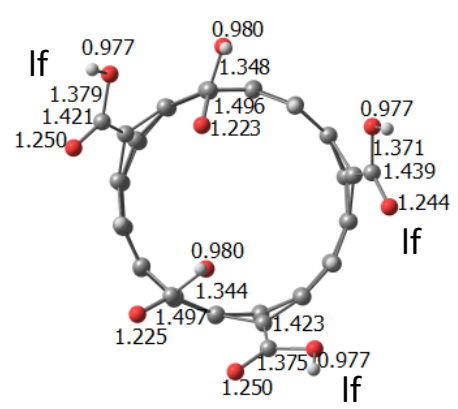

$(8,0)$-5a-water

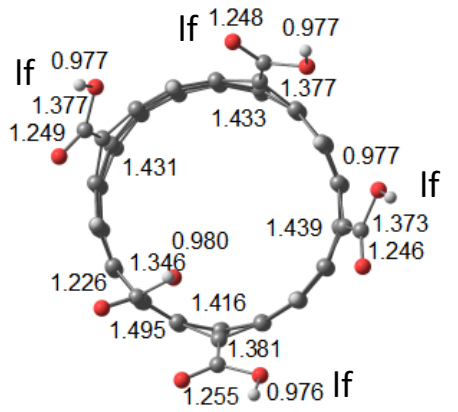

$(9,0)-5 a-w a t e r$

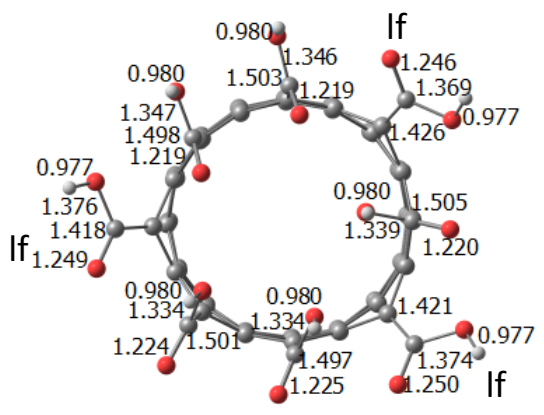

$(8,0)-8 a-w a t e r$

Fig. 2 - Optimized structures of $(4,4)-(\mathrm{COOH})_{n}$ and $(8,0)-(\mathrm{COOH})_{\mathrm{n}}(\mathrm{n}=5$ and 8$)$ in water. Distances are in $\AA$. Low-frequency $\mathrm{COOH}$ groups are marked by lf. Optimized structures in other solvents and in gas-phase are given in Figures $\mathrm{S} 3(\mathrm{n}=5)$ and $\mathrm{S} 4(\mathrm{n}=8)$. 


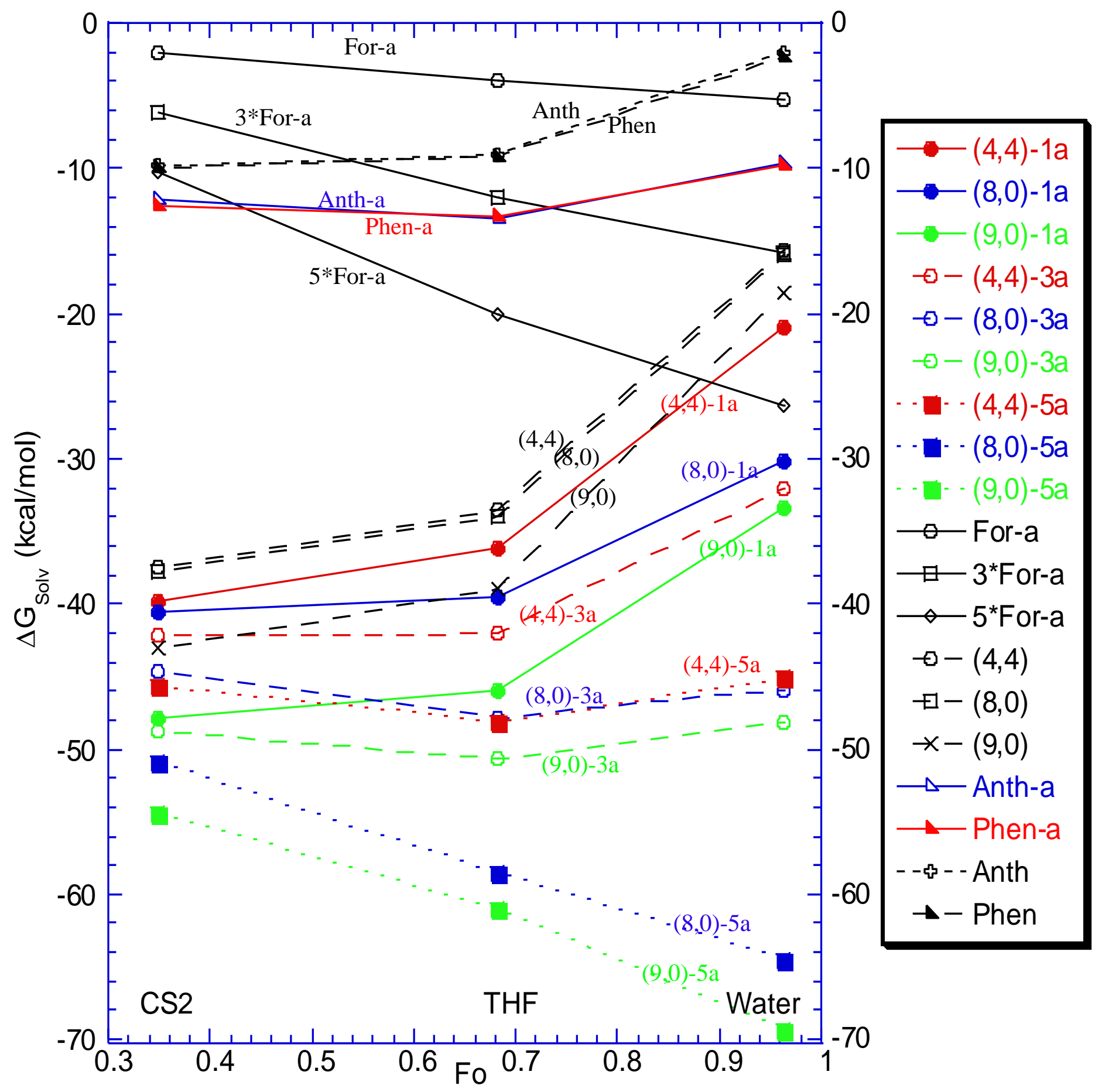

Fig. 3 - Variation of $\Delta \mathrm{G}_{\text {Solv }}$ of SWNT- $(\mathrm{COOH})_{\mathrm{n}}$ and simple organic acids in different medium. Onsager function $\mathrm{F}_{\mathrm{o}}=(\varepsilon-1) /(\varepsilon+2)$, where $\varepsilon$ refers to the dielectric constant of the medium. Ben, Anth, Phen and For stand for benzene, anthracene, phenanthrene and formic acid, respectively. 


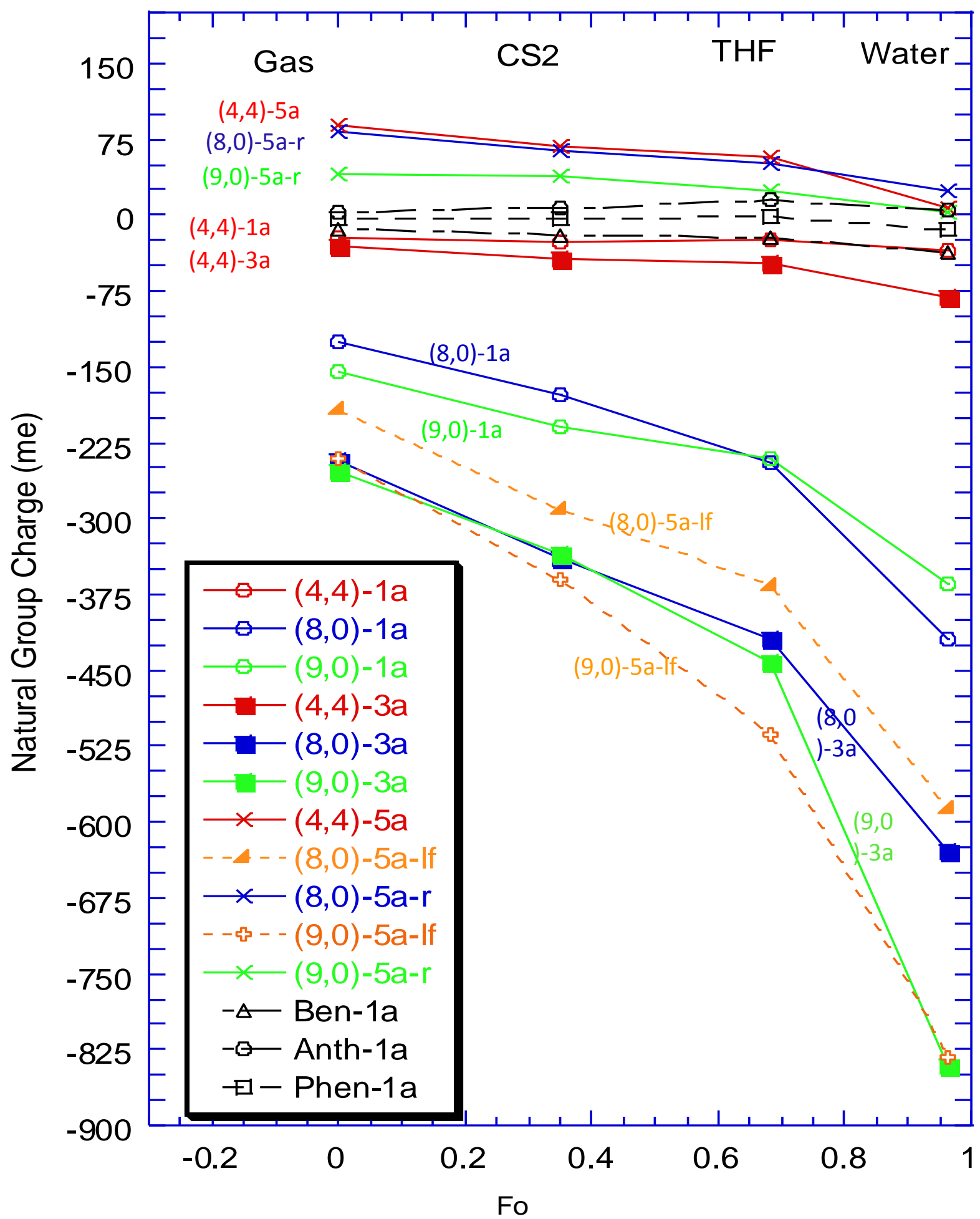

Fig. 4 - Variation of natural charge (q, me) of acid group of SWNT- $(\mathrm{COOH})_{\mathrm{n}}(\mathrm{n}=1,3$ and 5) and simple organic acids. If and $r$ stand for low-frequency and regular $\mathrm{COOH}$ group, respectively. 

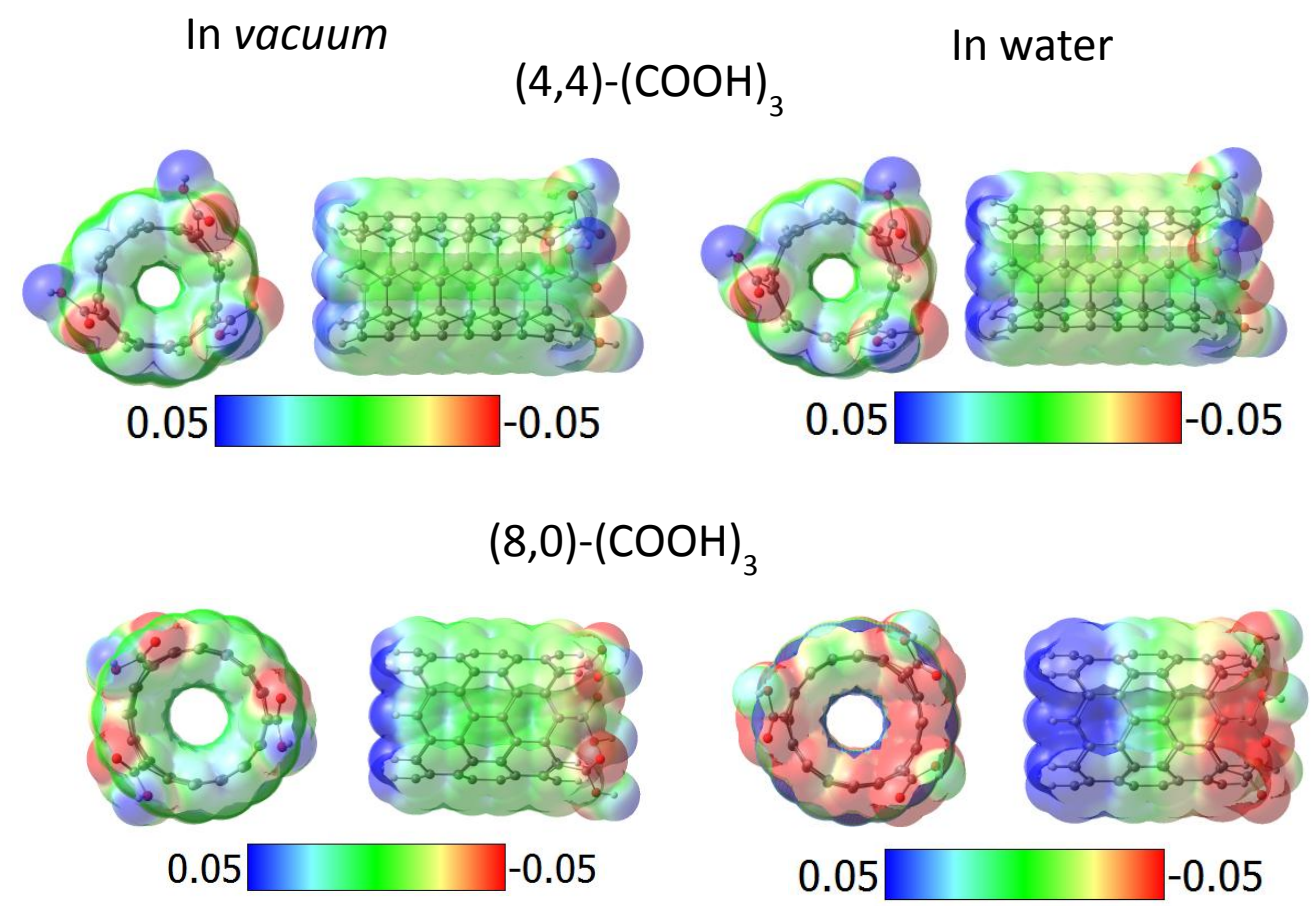

$(9,0)-(\mathrm{COOH})_{3}$
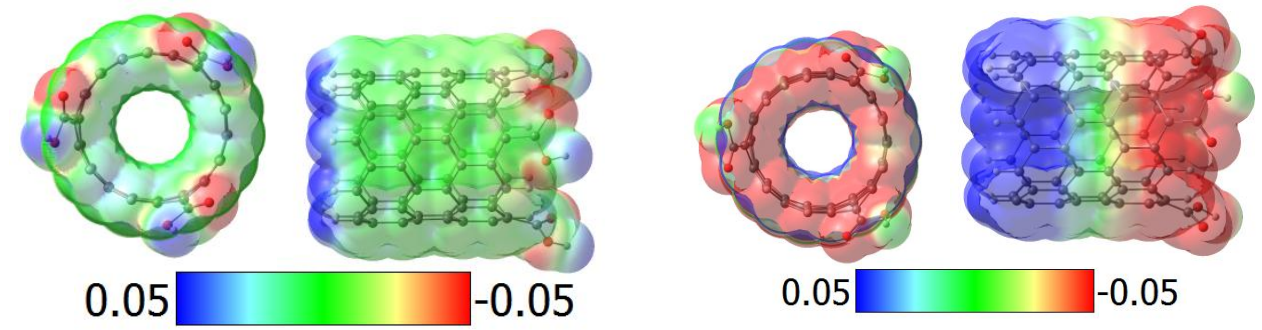

Fig. 5 - Top and side view of the molecular electrostatic potentials (MEP) of different SWNT- $(\mathrm{COOH})_{3}$ in gas-phase and in water. 


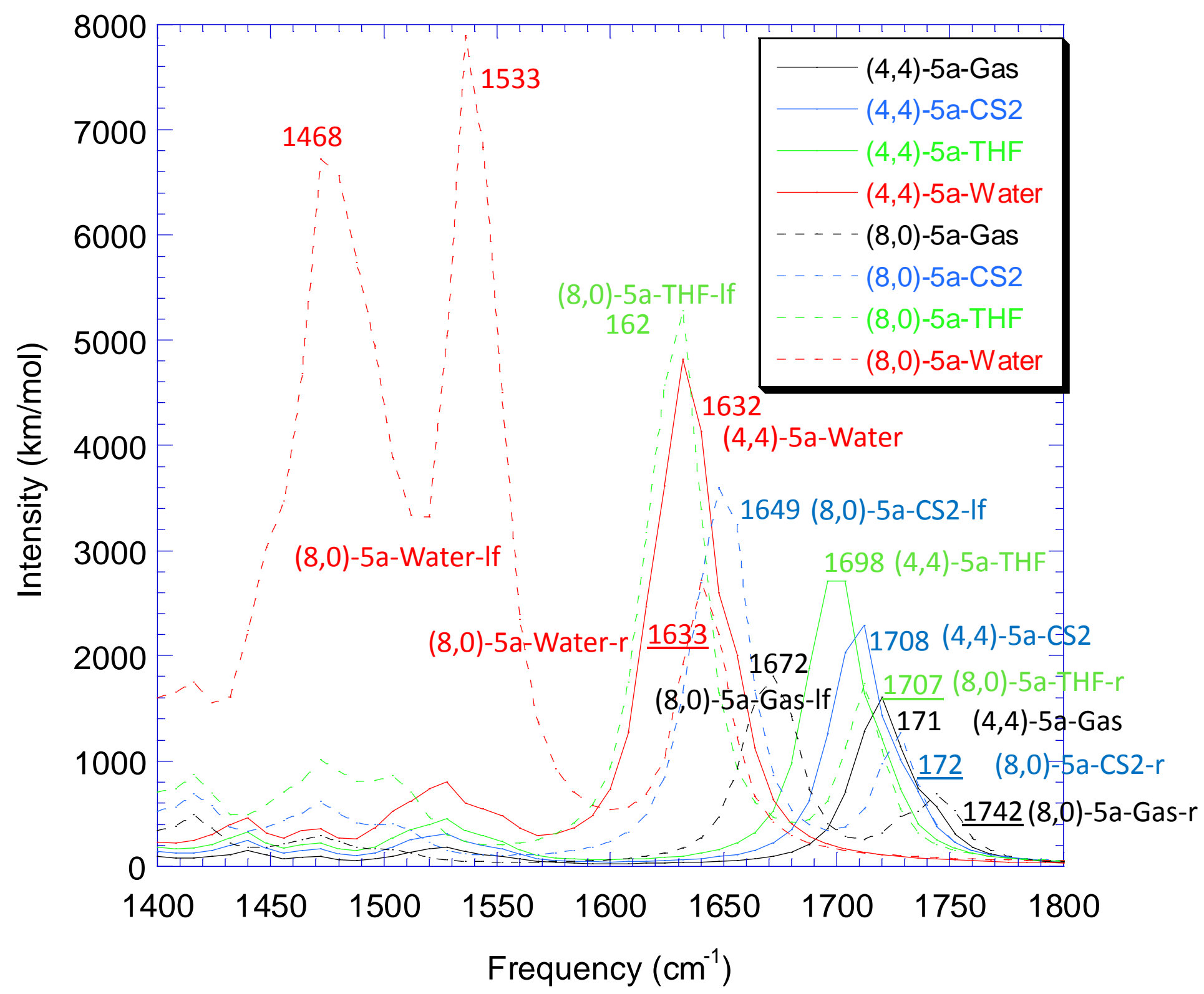

Fig. 6 - B3LYP IR spectra of $(4,4)-(\mathrm{COOH})_{5}$ and $(8,0)-(\mathrm{COOH})_{5}$ in different medium. FWHF is considered at $20 \mathrm{~cm}^{-1}$. Theoretical frequencies are scaled by 0.96 . Underlined values are from regular $\mathrm{COOH}(\mathrm{r}-\mathrm{COOH})$ group of zigzag tube. 


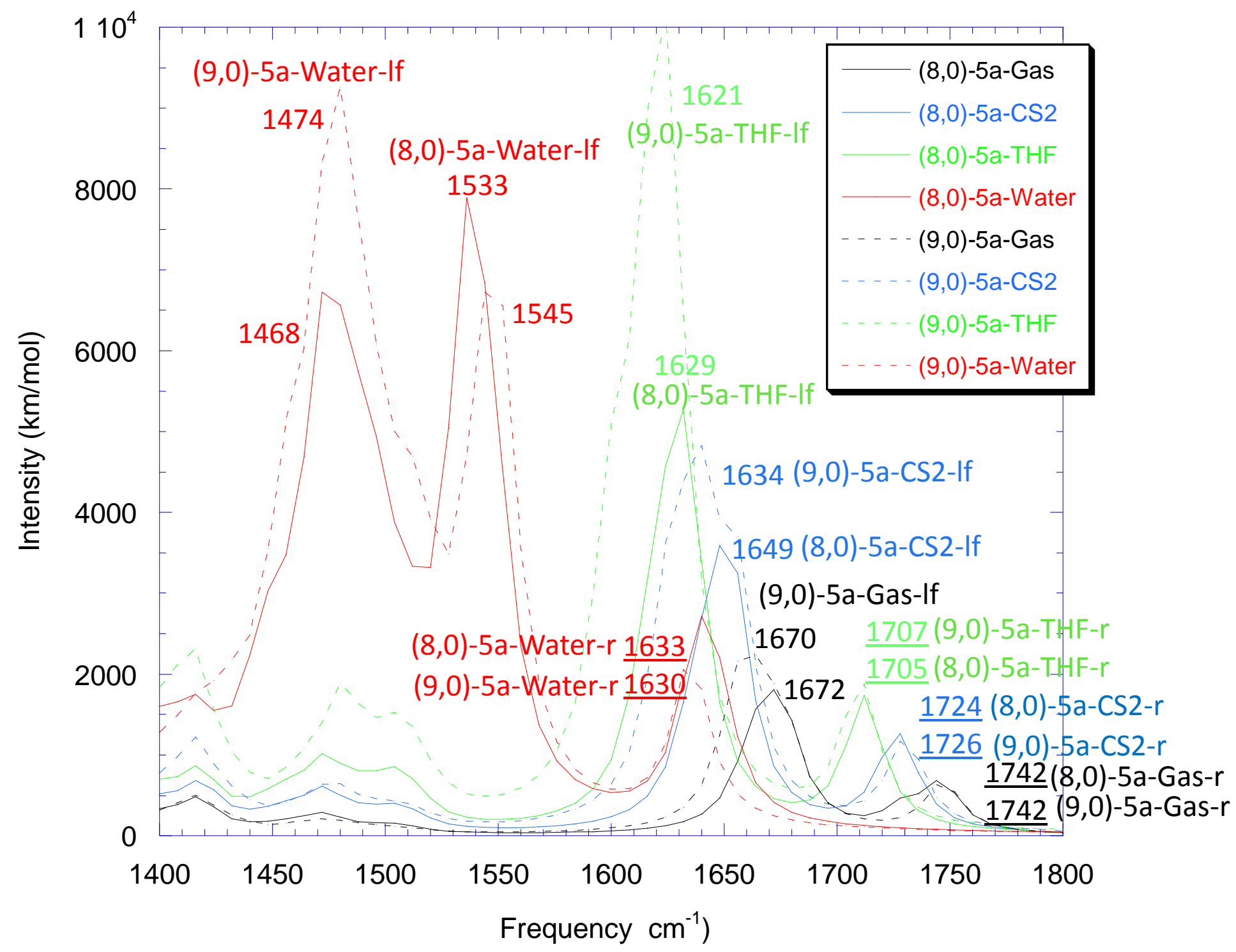

Fig. 7 - B3LYP IR spectra of $(8,0)-(\mathrm{COOH})_{5}$ and $(9,0)-(\mathrm{COOH})_{5}$ in different medium. FWHF is considered at $20 \mathrm{~cm}^{-1}$. Theoretical frequencies are scaled by 0.96 . Underlined values are from regular $\mathrm{COOH}$ group. 

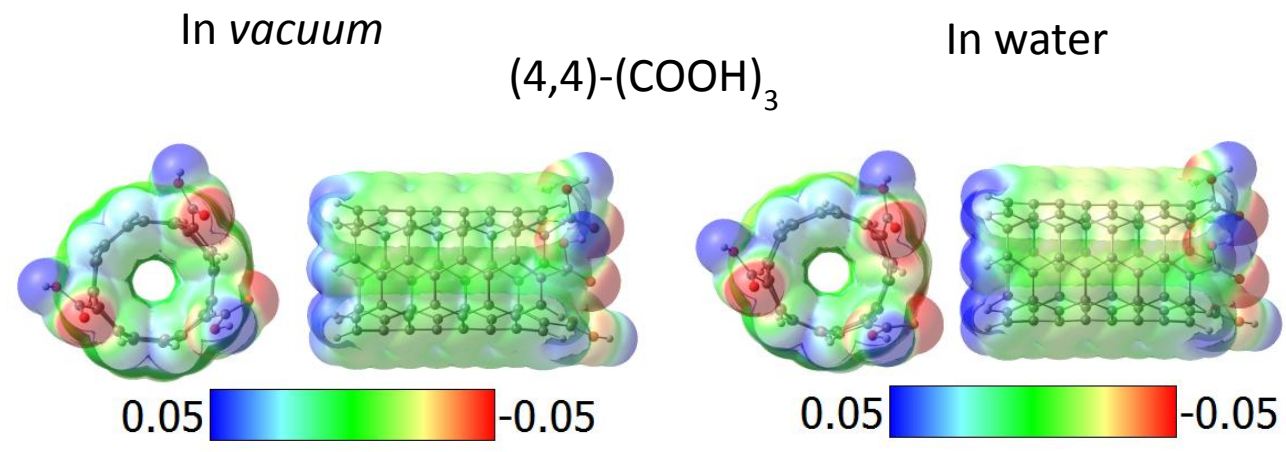

$(8,0)-(\mathrm{COOH})_{3}$
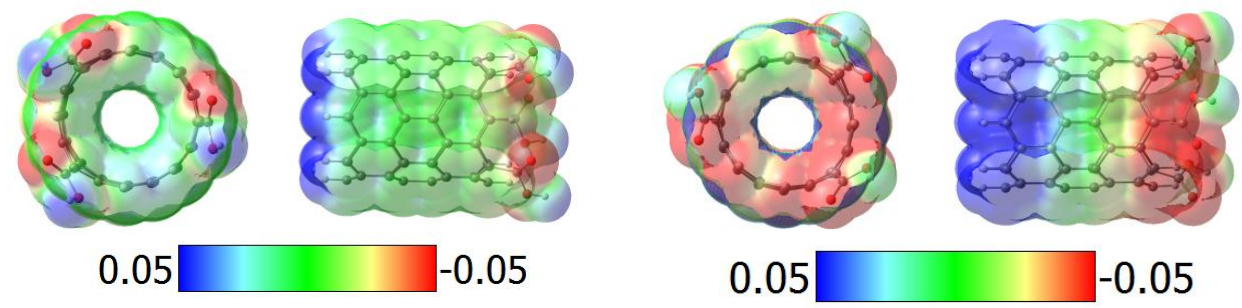

Top and side view of the molecular electrostatic potentials (MEP) of different $(4,4)-(\mathrm{COOH})_{3}$ and $(8,0)$ $(\mathrm{COOH})_{3}$ in vacuum and in water. Blue and red regions in MEP maps indicate positive and negative potential, respectively. Intermediate potentials are shown in green and yellow colors. No noticeable changes in MEP are found when the carboxylated $(4,4)$ tube is placed in water. However, differences are prominent for zigzag tubes in water. 
Supplementary Material
Click here to download Supplementary Material: SuppDoc.docx

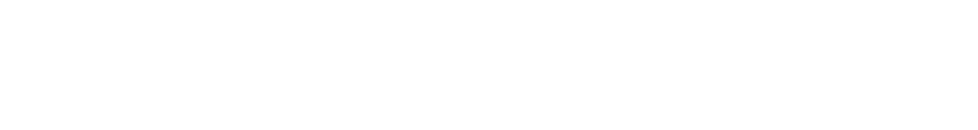
(1) . . . . . . . . . . . . . . . 